\title{
SHALL WE OBSERVE A RADIO BINARY PULSAR IN SN1987A?
}

\author{
QIAO G. J. \\ Center of Astronomy and Astrophysics, CCTS (World Laboratory) and Department of Geophysics, Peking University
}

\begin{abstract}
An induced-collapse model [hereafter IC model; He et al. (1990)] can overcome the problems of the single star model of SN1987A. According to the IC model, there is a possibility that the SN1987A remnant will be a binary system with two neutron stars, one of them (SK-69 202) will have a strong magnetic field and a high surface temperature which favors detection as an X-ray or $\gamma$-ray pulsar. If the surface temperature of the neutron star cools down to $T=10^{7} \mathrm{~K}$, a radio binary pulsar is expected. There is also the possibility that an X-ray or $\gamma$-ray pulsar will be observed first, and only later will a radio pulsar will be detected.

A newly formed neutron star is thought to have a short (millisecond) period. In this case, the core emission bearn is then very large (Qiao 1992) and is thus very likely to swing in the direction of the Earth.
\end{abstract}

\section{Introduction}

Models of SN1987A can be classified into two types, one in which the progenitor star was a single star (single star model; hereafter SS model), and another in which the progenitor star was in a binary system (binary star model; BS model).

The SS model has been studied widely and in detail [see Arnett et al. (1989), Nomoto et al. (1988)]. These models can provide explanations of the progenitor's evolution, explosive nucleosynthesis, optical, X-ray and $\gamma$-ray light curves, and dust formation. There are, however, yet some problems with the SS model, such as the hydrogen mixed into the very deep interior and the photoelectric opacity in fitting the X-ray and $\gamma$-ray light curves (see below).

The foregoing problems can be resolved easily in the IC model, which is one type of BS model but is very different from the usual BS model. In the usual BS model only one component of the binary exploded. But in the IC model, both stars exploded, one after the other. The later explosion (SK-69 202, hereafter star B) was induced by the first explosion (the companion of SK-69202, hereafter, star A).

If the IC model is correct, we would expect to observe an X-ray and/or $\gamma$-ray binary pulsar and a binary radio pulsar in SN1987A.

\section{Problems with the SS model}

1. A hydrogen-rich envelope mixed into a very deep layer. One of the surprises associated with SN1987A has been observational evidence indicating that very deep and extensive "mixing" has occurred. For model $14 \mathrm{E} 1$, the hydrogen recombination front reaches $M_{\mathrm{r}}=3.6 \mathrm{M}_{\odot}($ at $t=80 \mathrm{~d})$ and
$2.0 \mathrm{M}_{\odot}($ at $100 \mathrm{~d})$, which indicates the existence of hydrogen in the deep interior.

The existence of such mixing may come from the Rayleigh-Taylor instability. A three dimensional simulation shows compositional mixing and density contrasts of approximately a factor of 8 (Nomoto $e t$ al. 1988). So the problem is open.

2. Fitting the light curves of the hard $\mathrm{X}$-rays and $\gamma$-rays. The light curves of hard X-rays and $\gamma$-rays cannot be fitted in the usual model; good agreement appears only in calculations for a model with the photoelectric opacity reduced by a factor of 9 at $M_{\mathrm{r}}<6 \mathrm{M}_{\odot}$. The spectrum at $20-30 \mathrm{keV}$ depends on the clumpiness observations (Nomoto et al. 1988) which are very favorable to IC model.

\section{The IC model}

In the induced collapse model, the progenitor of SN1987A is a binary system, the two stars exploded one after another. SK-69202 was induced to collapse by the explosion of its companion (star A). In this case, the hydrogen-rich envelope of star B should be mixed down to the core, and the elements mixed into the hydrogen-rich envelope in a "clumpy" manner which implies that a large fraction of the X-rays could be transported through the hydrogen and helium-rich regions without suffering much photoelectric absorption, thus effectively reducing the opacity (He et al. 1990).

\section{Final resulting objects in SN1987A}

In the IC model, two stars exploded, and the change of the separation $a$ can be written (Savonije 1983) 
Table 1

\begin{tabular}{|c|c|c|c|c|c|c|c|}
\hline \multicolumn{3}{|c|}{ Initial binary system } & \multicolumn{2}{|c|}{ Intermediate state } & \multicolumn{3}{|c|}{ Resulting objects } \\
\hline $\begin{array}{c}M_{1}+M_{2} \\
\left(\mathrm{M}_{\odot}\right)\end{array}$ & $\begin{array}{c}a \\
(\mathrm{~cm})\end{array}$ & $\begin{array}{l}\begin{array}{l}\text { Period } \\
\text { (days) }\end{array} \\
\end{array}$ & $\begin{array}{c}M_{1}+\bar{M}_{2} \\
\left(\mathrm{M}_{\odot}\right)\end{array}$ & $\begin{array}{c}a \\
(\mathrm{~cm})\end{array}$ & $\begin{array}{c}M_{1}+M_{2} \\
\left(M_{\odot}\right)\end{array}$ & $\begin{array}{c}a \\
(\mathrm{~cm})\end{array}$ & $\begin{array}{l}\text { Period } \\
\text { (hours) }\end{array}$ \\
\hline & & & & & & $3.7 \times 10^{11}$ & 22.6 \\
\hline $8+16$ & $4 \times 10^{12}$ & 10.3 & $0.8+16$ & $2.8 \times 10^{12}$ & $0.8+1.4$ & $4.2 \times 10^{10}$ & 0.86 \\
\hline & & & & & & $4.3 \times 10^{11}$ & 22.7 \\
\hline $8+16$ & $4 \times 10^{12}$ & 10.3 & $1.2+16$ & $2.9 \times 10^{12}$ & $1.2+1.4$ & $1.1 \times 10^{10}$ & 0.11 \\
\hline $4+16$ & $4 \times 10^{12}$ & 11.3 & $0.8+16$ & $3.4 \times 10^{12}$ & $0.8+1.4$ & $5.0 \times 10^{10}$ & $\begin{array}{l}29.7 \\
1.13\end{array}$ \\
\hline
\end{tabular}

as

$$
\frac{a^{\prime}-a}{a}=-2\left\{1+(\alpha-1) \frac{M_{1}}{M_{2}}-\frac{\alpha}{2}\left(\frac{M_{1}}{M}\right)\right\} \frac{\Delta M_{1}}{M_{1}}+2 \frac{\Delta J_{\text {orb }}}{J_{\text {orb }}},
$$

where $M_{1} \quad\left(M_{2}\right)$ is the maiss of star B (star A), $\Delta M_{2}=-\Delta M_{1}+\delta M, \alpha=\delta M / M_{1}$, $J_{\text {orb }}=\left(M_{1} M_{2} / M\right) \Omega a^{2}, p$ is the orbital period of the binary, and $M=M_{1}+M_{2}, \Omega=2 \pi / p$.

$$
\Delta J_{\text {orb }}=-J_{\text {orb }}\left[1-\frac{M_{1}^{\prime}}{M_{1}}\left(\frac{M}{M^{\prime}} \frac{a^{\prime}}{a}\right)^{\frac{1}{2}}\right],
$$

here $M_{1}^{\prime}$ is the mass of star B after the explosion, so $M^{\prime}=M_{1}^{\prime}+M_{2}^{\prime}$. In our case $\Delta M_{2}=0$ and $\alpha=1$, thus we have

$$
\frac{a^{\prime}-a}{a}=-2\left(1-\frac{M_{1}}{2 M}\right) \frac{\Delta M_{1}}{M_{1}}-2(1-\xi) \xi=\frac{M_{1}^{\prime}}{M_{1}}\left(\frac{M a^{\prime}}{M^{\prime} a}\right)^{\frac{1}{2}}
$$

and

$$
x^{2}-2 \frac{M_{1}^{\prime}}{M_{1}}\left(\frac{M}{M^{\prime}}\right)^{\frac{1}{2}} x+\left[1-2\left(1-\frac{M_{1}}{2 M}\right) \frac{\left|\Delta M_{1}\right|}{M_{1}}\right]=0,
$$

where $x=\left(a^{\prime} / a\right)^{\frac{1}{2}}$.

After two explosions, the system either becomes a binary with two neutron stars or the system is disrupted. The parameters of the binary system before the explosions as well as the objects after the explosions are shown in table 1.

From table 1 we can see that the final objects have masses of $0.8-1.2 \mathrm{M}_{\odot}$ and $1.4 \mathrm{M}_{\odot}($ star $B)$ and a $\sim 1-24$ hour orbital period.

\section{Shall we observe an X-ray and/or $\gamma$-ray binary pulsar?}

Under the influence of the explosion of star B, the neutron star formed from star A should lose its magnetic and rotational energy and become a neutron star with a weaker magnetic field and a longer rotational period. But the newly formed neutron star (formed from star B) should have a stronger magnetic field. In this strong magnetic field, inverse Compton scattering (ICS) is a more important source of X-rays and $\gamma$-rays (Xia et al. 1985, Daugherty and Harding 1989). This makes the polar cap much brighter in $\mathrm{X}$-rays and $\gamma$-rays.

Let us consider the ICS of the thermal photons $\omega$ with the pairs in the polar-cap region. The outgoing photons $\omega^{\prime}$ resulting from the scattering can be described (Qiao et al. 1986) as

$\hbar \omega^{\prime}=\frac{1+\beta}{2} \gamma \frac{\hbar^{2} \omega^{2} \sin ^{2} \theta+2 \gamma m c^{2} \hbar \omega(1-\beta \cos \theta)}{m c^{2}+\gamma \hbar \omega(1+\beta)(1-\cos \theta)}$

for $\theta^{\prime}=0$, where $\theta\left(\theta^{\prime}\right)$ is the angle between the direction of the incoming (or outgoing ) photons and the magnetic field, and $m$ is the rest mass of an electron. If the surface temperature of the neutron star $T=10^{7} \mathrm{~K}$, we have $\gamma \leq 10^{2}$ (Xia et al. 1985). For a black body peak frequency $\omega_{\mathrm{m}}=3.8 \times 10^{11} \mathrm{~T}$, we get $2 \mathrm{keV} \leq \hbar \theta^{\prime} \leq 8 \times 10^{4} \mathrm{keV}$ (for $0 \leq \theta \leq \pi$, $\left.\gamma \leq 10^{2}\right)$.

In this case, we might expect to observe an Xray and/or $\gamma$-ray pulsar. When the rotational period is in the millisecond range, the central beam (i.e., the core beam) is much wider (Qiao 1992), which increases the probability that it will be observed from the Earth.

\section{Shall we observe a radio binary pulsar in SN1987A?}

In the radio band, $\gamma \hbar \omega \ll m c^{2}$. Eq.(5) then becomes

$$
\omega^{\prime}=2 \gamma^{2} \omega(1-\beta \cos \theta)
$$

As $\gamma \approx 10^{2}, 30^{\circ} \leq \theta \leq 60^{\circ}$, we get $0.27 \mathrm{GHz} \leq$ $f^{\prime} \leq 1 \mathrm{GHz}$. In this case, a radio pulsar will be observed (for suitable magnetic inclination $\alpha$ and impact angle $\beta$ (see Qiao 1991). But if the surface temperature of the neutron star is high enough, for example, $T>5 \times 10^{6} \mathrm{~K}$, and the gap is high enough, $\gamma<10$, no radio emission will be observed. 


\section{Conclusions}

1. The characteristics of the newly formed neutron star in SN1987A favors the possibility that it will be observed as an X-ray and/or $\gamma$-ray pulsar.

2. If the surface temperature of the neutron star is not high enough, we will observe a radio pulsar. However, if the temperature is high enough, it will be difficult to observe in the radio band. If we search in the radio band, lower frequency emission is favored (because $\gamma$ is small).
3. If we find a pulsar in SN1987A, there is a possibility that it will be a binary pulsar system with two neutron stars. If this is the case, it appears that a new mechanism has resulted in the formation of this binary pulsar system.

Acknowledgments: We are grateful to Professors Z. W. Li and Y. Q. Ma for helpful discussions. We thank Mr. S. B. Deng for help with the calculations. This project was supported by the Natural Science Foundation of China. 